\title{
Processes in the magnetized chromosphere of the Sun
}

\author{
S. S. Hasan \\ Indian Institute of Astrophysics, Koramangala, Bangalore 560034, India \\ email: hasan@iiap.res.in
}

\begin{abstract}
We review physical processes in magnetized chromospheres on the Sun. In the quiet chromosphere, it is useful to distinguish between the magnetic network on the boundaries of supergranules, where strong magnetic fields are organized in mainly vertical flux tubes and internetwork regions in the cell interiors, which have traditionally been associated with weak magnetic fields. Recent observations from Hinode, however, suggest that there is a significant amount of horizontal magnetic flux in the cell interior with large field strength. Furthermore, processes that heat the magnetic network have not been fully identified. Is the network heated by wave dissipation and if so, what is the nature of these waves? These and other aspects related to the role of spicules will also be highlighted. A critical assessment will be made on the challenges facing theory and observations, particularly in light of the new space experiments and the planned ground facilities.
\end{abstract}

Keywords. Sun: magnetic - magnetohydrodynamics (MHD), Sun: chromosphere, Sun: oscillations

\section{Introduction}

It is well known that magnetic fields play an important role in the dynamics of the solar chromosphere. In the chromosphere on the quiet Sun it is useful to distinguish between the magnetic network on the boundary of supergranulation cells (Simon \& Leighton 1964), where strong magnetic fields are organized in mainly vertical flux tubes, and internetwork regions in the cell interiors, where it was earlier believed that the magnetic fields are weaker and dynamically less important. As we shall subsequently see, this picture needs to be modified in the light of new observations.

The main focus of this review is a study of dynamical processes in the magnetized solar chromosphere, which we treat as the region above the photospheric surface (defined as the layer with continuum optical depth unity) with an extension of about $2 \mathrm{Mm}$. Above the temperature minimum and up to the middle chromosphere (close to a height of about $1.5 \mathrm{Mm}$ ), the atmosphere can be effectively regarded as almost isothermal. In the higher layers, the observed properties of the chromosphere are strongly influenced by magnetic fields. A convenient way to parameterize the field strength is in terms of $\beta$, defined as the ratio of the gas to magnetic pressure. The $\beta=1$ (which is not uniform with height) provides a natural separation of the atmosphere into magnetic and non-magnetic (or weakly magnetized) regions. In the lower chromosphere and below, the magnetic field is structured in the form of magnetic flux tubes, which occur at the cell boundaries and constitute the well known magnetic network. These tubes that are mainly vertical and in pressure equilibrium with the outside medium expand upward to conserve magnetic flux. From a low filling factor $(<1 \%)$ in the photosphere the tubes spread to $15 \%$ in the layers of formation of the emission features in the $\mathrm{H}$ and $\mathrm{K}$ lines of ionized calcium (at a height of $1 \mathrm{Mm}$ ) and to $100 \%$ in the so-called magnetic canopy. The remaining quiet Sun outside the network is called the internetwork, sometimes also referred to as cell interior. 
The canonical picture of the magnetic network is that it consists of vertical magnetic fields clumped into elements or flux tubes that are located in intergranular lanes, have magnetic field strengths in the kilogauss range, and have diameters of the order of $100 \mathrm{~km}$ or less at their footpoints in the photosphere (e.g., Gaizauskas 1985; Zwaan 1987). These magnetic elements can be identified with bright points seen in images taken in the G-band $(430.5 \mathrm{~nm})$. High resolution observations show that these flux elements are in a highly dynamical state due to buffeting by convective flows on granular and supergranular scales (e.g., Muller et al. 1994; Berger \& Title 1996; Nisenson et al. 2003). With the availability of new ground-based telescopes at excellent sites and sophisticated image reconstruction techniques it is now possible to examine magnetic elements with an improved resolution of about $0.17^{\prime \prime}$ and investigate their structure and dynamics in unprecedented detail (e.g., Berger et al. 2004,; Rouppe van der Voort 2005; Langangen et al. 2007). High-quality observations of photospheric magnetic fields are now also being obtained with the Solar Optical Telescope (SOT) on Hinode (e.g., Lites et al. 2007, 2008). Magnetoconvection models have been developed to understand the three-dimensional structure and evolution of the magnetic field and its interactions with convective flows (e.g., Vögler et al. 2005; Schaffenberger et al. 2006; Steiner et al. 2008).

The chromospheric network is most clearly seen in filter images taken in the Ca II H \& K lines (e.g.,Gaizauskas 1985; Rutten 2007) and in the Ca II IR triplet (Cauzzi et al. 2008). In $\mathrm{H}$ or $\mathrm{K}$ line images the network shows up as a collection of "coarse mottles" or "network grains" that stand out against the darker background. The network grains are continuously bright with intensities that vary slowly in time, in contrast to the "fine mottles" or "cell grains" which are located in the cell interiors and are much more dynamic (e.g., Rutten and Uitenbroek 1991).

Some of the important questions that need to be addressed are: (a) What are the physical processes contributing to the dynamics and heating of the magnetic network and produce the observed enhanced calcium emission? (b) What is the nature of the magnetic field in the internetwork and are these fields dynamically important?and (c) What mechanisms contribute to the fine structure of the chromosphere such as spicules? We shall attempt to shed light on these questions. The plan of this review is as follows: in Sect. 2, we briefly discuss recent observations of the magnetic network, followed in Sect. 3 by a theoretical model for interpreting the chromospheric emission in terms of magnetoacoustic shocks. In Sect. 4, we examine the nature of the internetwork field, particularly in the light of recent observations from Hinode. In Sect. 5, we discuss spicules and their role in the dynamics and energetics of the chromosphere. Finally in Sect. 6, we consider some implications and outstanding issues, particularly for future observational ground and space programmes.

\section{Observations of the magnetic network}

Recently a large number of high-resolution images of the solar atmosphere have become available from gound-based telescope, thanks largely to adaptive optics and image reconstruction techniques. These support the hypothesis of a network patch consisting of several discrete magnetic elements. A comparison with the Ca II line center image shows that the excess chromospheric emission is localized directly above the photospheric flux tubes, although the bright features seen in Ca II are more diffuse than those seen in the G-band. Time sequences of such images show that the chromospheric network is contin-

ually bright (e.g., Tritschler et al. 2007). Rutten (2006, 2007) presented reviews and a synthesis of recent high-resolution observations of the solar chromosphere. In the Ca II H \& K lines, the network shows up as a collection of "Ca II bright points" or "grains". He 


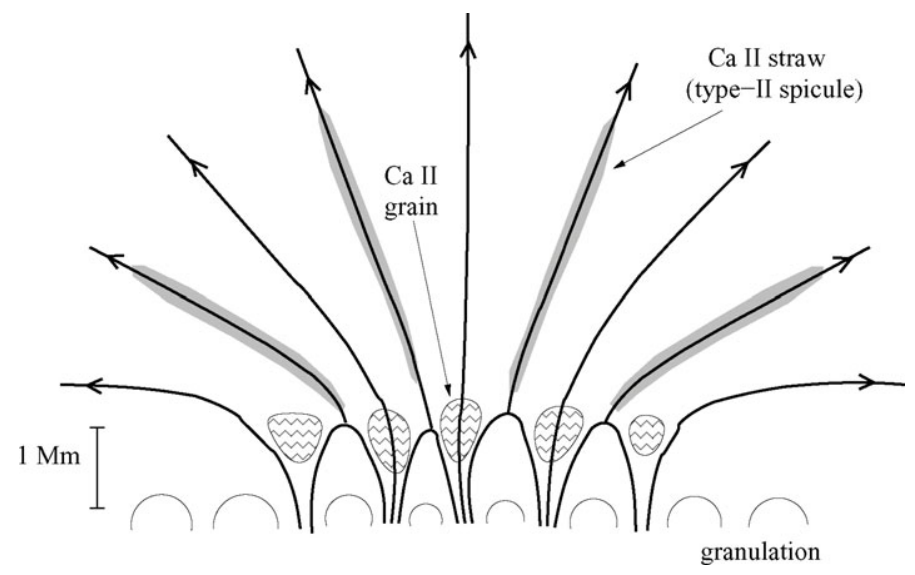

Figure 1. Schematic diagram showing the structure of a magnetic network element on the quiet Sun. The thin half-circles at the bottom of the figure represent the granulation flow field, and the thick curves represent magnetic field lines of flux tubes that are rooted in the intergranular lanes. The Ca II bright grains are thought to be located inside the flux tubes at heights of about $1 \mathrm{Mm}$ above the base of the photosphere. We suggest that the Ca II "straws" (Rutten 2006) may be located at the boundaries between the flux tubes (from Hasan \& van Ballegoiijen 2008).

also identifies an exciting new phenomenon called "straws" that extend radially outward from network bright points (see Figure 8 in Rutten 2007). These straws are very thin, occur in "hedge rows", and are very short-lived (10-20 s). They appear to be closely related to the so-called "type-II" spicules recently identified in limb observations with SOT on Hinode (De Pontieu 2007a; see Sect. 5 for more details).

Our interpretation of the Ca II observations is summarized in Figure 1, where we show a vertical cross section of a magnetic network element consisting of several discrete flux tubes. We suggest that the Ca II network grains are located inside the magnetic flux tubes, and give rise to the bulk of the Ca II emission from the network element. The grains are thought to be located at heights between $500 \mathrm{~km}$ and $1500 \mathrm{~km}$ above the photosphere where the flux tubes are no longer "thin" compared to the pressure scale height (about $200 \mathrm{~km}$ ), but are still well separated from each other. The Ca II straws ("type-II" spicules) have widths of order $100 \mathrm{~km}$, and are located at larger heights (several Mm) where the widths of the flux tubes are much larger than $100 \mathrm{~km}$. Therefore, we suggest the straws are not directly associated with the network grains in the low chromosphere. In Figure 2 we assumed that the straws ("type-II" spicules) are located at the interfaces between the flux tubes, as suggested by van Ballegoiijen \& Nisenson (1998).

It seems unlikely that long-period waves are also responsible for the heating of the Ca II network grains in the low chromosphere. Simulations of shock waves with periods $P \sim 200$ s in a plane-parallel, non-magnetic atmosphere have shown that such waves produce large asymmetries in the Ca II H line profiles, and strong variations in the integrated emission. If the grains were heated by such long-period waves, they should exhibit similar strong intensity variations. This is not observed, so the long-period waves observed in network elements cannot be the main source of heating for the Ca II grains. Network grains could possibly be heated by dissipation of waves with shorter periods $(P<100 \mathrm{~s})$. Ground-based observations of high-frequency waves in small network elements are affected by seeing, so it is possible that waves with periods $P<100$ s do exist in network elements but are simply not observable from the ground. This hypothesis could be tested using Ca II H images from Hinode (Kosugi et al. 2007), keeping in mind of course that the passband of the $\mathrm{Ca}$ II $\mathrm{H}$ filter on SOT also includes a significant photospheric 

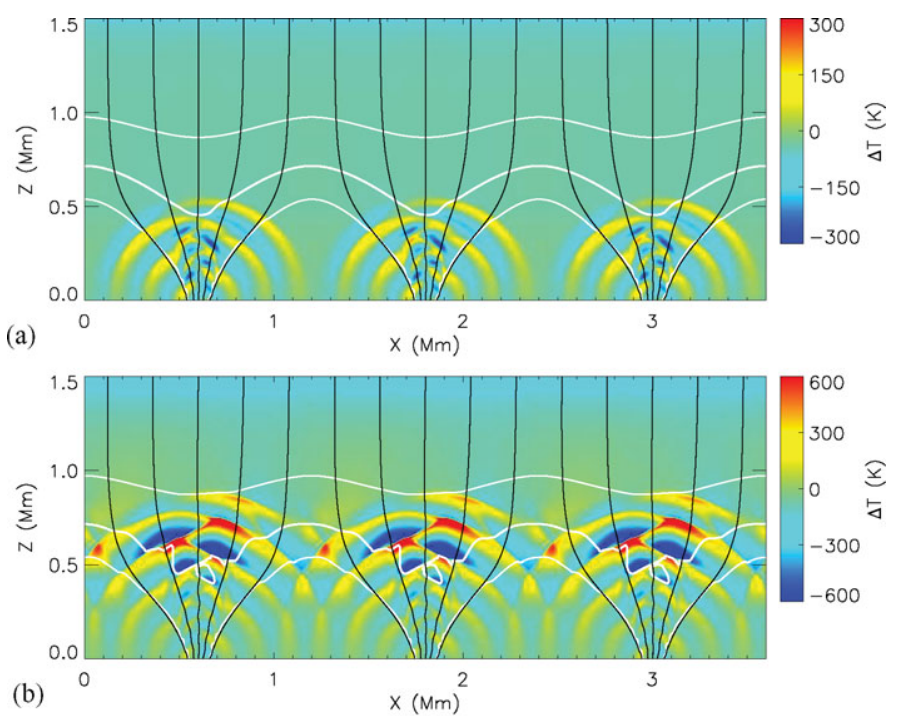

(b)

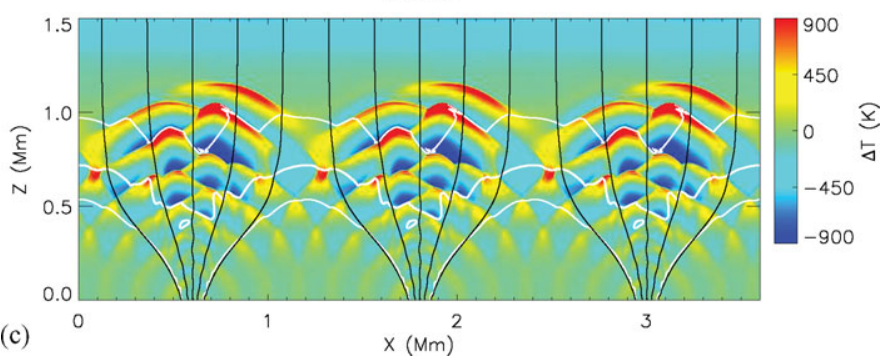

Figure 2. The temperature perturbation, $\Delta T$, (about the initial state) at (a) $75 \mathrm{~s}$, (b) $122 \mathrm{~s}$, and (c) $153 \mathrm{~s}$ in a network region consisting of 3 flux tubes. Wave excitation is due to periodic horizontal motion at the lower boundary, with an amplitude of $750 \mathrm{~m} \mathrm{~s}^{-1}$, and a period of $24 \mathrm{~s}$. The black curves denote the magnetic field lines, and the color scale shows the temperature perturbation. The white curves denote contours of constant $\beta$ corresponding to $\beta=0.1$ (upper curve), 1.0 (thick curve) and 10 (lower curve) (from Hasan \& van Ballegoiijen 2008).

contribution. Alternatively, network grains could be heated by dissipation of Alfvén waves. At present there is no direct observational evidence for Alfvén waves in flux elements in the photosphere, nor at heights where the Ca II H line is formed.

\section{Dynamics of the magnetic network}

Earlier work idealized the network in terms of thin flux tubes (e.g., Roberts \& Webb 1978) and treated wave propagation in terms of the well known transverse (kink) and longitudinal (sausage) modes (e.g., Spruit 1981). Several investigations have focused on the generation and propagation of transverse and longitudinal wave modes and their dissipation in the chromosphere (e.g., Zhugzhda et al. 1995; Ulmschneider 2003 and references therein). Torsional waves have received some attention (e.g., Hollweg et al. 1982; Routh, Musielak \& Hammer 2007). Hasan and Kalkofen (1999) examined the excitation of transverse and longitudinal waves in magnetic flux tubes by the impact of fast granules on flux tubes, as observed by Muller and Roudier (1992) and Muller et al. (1994), and following the investigation by Choudhuri et al. (1993), who studied the generation of kink waves by footpoint motion of flux tubes. The observational signature of the modelled process was highly intermittent in radiation emerging in the $\mathrm{H}$ and $\mathrm{K}$ lines, contrary to observations. 
By adding waves that were generated by high-frequency motions due to the turbulence of the medium surrounding flux tubes the energy injection into the gas inside a flux tube became less intermittent, and the time variation of the emergent radiation was in better agreement with the more steady observed intensity from the magnetic network (Hasan et al. 2000).

The above studies modelled wave excitation and propagation in terms of the KleinGordon equation, motivated by the identification of the power peak near $7 \mathrm{~min}$. in the observed power spectrum (Lites et al. 1993) with the cutoff period of kink waves in thin magnetic flux tubes (Kalkofen 1997). This analysis was based on a linear approximation in which the longitudinal and transverse waves are decoupled. However, the motions are expected to become supersonic higher up in the atmosphere. At such heights, nonlinear effects become important, leading to a coupling between the transverse and longitudinal modes. Some progress on this question has been made in one dimension, using the nonlinear equations for a thin flux tube by Ulmschneider et al. (1991), Huang et al. (1995), Zhugzhda et al. (1995), and more recently by Hasan et al. (2003) and Hasan \& Ulmschneider (2004), who examined mode coupling between transverse and longitudinal modes in the magnetic network. By solving the nonlinear, time-dependent MHD equations it was found that significant longitudinal wave generation occurs in the photosphere, typically for Mach numbers as low as 0.2 , and that the onset of shock formation occurs at heights of about $600 \mathrm{~km}$ above the photospheric base, accompanied by heating (Hasan et al. 2003, Huang et al. 1995). The efficiency of mode coupling was found to depend on the magnetic field strength in the network and achieved a maximum for field strengths corresponding to $\beta \approx 0.2$, when the kink and tube wave speeds are almost identical. This can have interesting observational implications. Furthermore, even when the two speeds are different, once shock formation occurs, the longitudinal and transverse shocks exhibit strong mode coupling.

The above studies on the magnetic network make use of two important idealizations: they assume that the magnetic flux tubes are thin, an approximation that becomes invalid at about the height of formation of the emission peaks in the cores of the $\mathrm{H}$ and $\mathrm{K}$ lines; and they neglect the interaction of neighboring flux tubes. Some progress in this direction has been made in recent years by Rosenthal et al. (2002) and Bogdan et al. (2003), who studied wave propagation in a two-dimensional stratified atmosphere, assuming a potential magnetic field to model the network and internetwork regions on the Sun. They examined the propagation of waves that are excited from a spatially localized source in the photosphere. Their results indicate that there is strong mode coupling between fast and slow waves at the so-called magnetic canopy, which they identify with regions where the magnetic and gas pressures are comparable. Wave propagation in a more realistic configuration consisting of a flux sheet embedded in a field free atmosphere was considered by Hasan et al. (2005) and in multiple flux sheets by Hasan \& van Ballegoiijen (2008). Other related work has been carried out using 3-D simulations by Vögler et al. (2005), Schaffenberger et al. (2005) and Carlsson and Bogdan (2006).

Figure 2 shows the wave propagation in a network element idealized in terms of three identical flux tubes (in a 2-D medium) driven by transverse periodic motions at the lower boundary with a velocity amplitude of $750 \mathrm{~m} \mathrm{~s}^{-1}$ and a period of $24 \mathrm{~s}$. The black and white lines respectively denote the magnetic field and contours of constant $\beta$ for values of $\beta=0.1$ (upper curve), 1.0 (thick curve) and 10 (lower curve). At the initial epoch (the equilibrium state), the magnetic field strength on the axis of the tubes at the base $z=0$ is $1000 \mathrm{G}$, corresponding to a $\beta$ of about 2.0 .

The horizontal motions at the lower boundary produce compressions and decompressions of the gas in the flux tube which generate an acoustic like wave (most effectively 
Table 1. Temporal maximum of horizontally averaged vertical component of fluxes (from Vigeesh et al. 2008).

\begin{tabular}{lrrrrr}
\hline & $\mathrm{F}_{A, z}\left(10^{6} \mathrm{erg} \mathrm{cm}^{-2} \mathrm{~s}^{-1}\right)$ & $\mathrm{F}_{P, z}\left(10^{6} \mathrm{erg} \mathrm{cm}^{-2} \mathrm{~s}^{-1}\right)$ \\
\cline { 2 - 6 } Initial Excitation & $z=100 \mathrm{~km}$ & $z=500 \mathrm{~km} \quad z=1000 \mathrm{~km} \quad z=100 \mathrm{~km} \quad z=500 \mathrm{~km} \quad z=1000 \mathrm{~km}$ \\
\hline $0.75 \mathrm{~km} \mathrm{~s}^{-1}, 24 \mathrm{~s}$ & 11.36 & 1.96 & 1.33 & 29.38 & 1.08 \\
$0.75 \mathrm{~km} \mathrm{~s}^{-1}, 120 \mathrm{~s}$ & 35.75 & 27.70 & 4.02 & 134.29 & 0.79 \\
$0.75 \mathrm{~km} \mathrm{~s}^{-1}, 240 \mathrm{~s}$ & 20.90 & 8.58 & 3.30 & 131.84 & 0.36 \\
$1.50 \mathrm{~km} \mathrm{~s}^{-1}, 24 \mathrm{~s}$ & 44.55 & 7.68 & 3.34 & 115.79 & 4.29 \\
$3.00 \mathrm{~km} \mathrm{~s}^{-1}, 24 \mathrm{~s}$ & 168.41 & 30.40 & 6.22 & 434.03 & 0.07 \\
\hline
\end{tabular}

at the interface between the tube and ambient medium as shown by Hasan et al. 2005) that propagates isotropically with an almost constant sound speed. This can be discerned from the almost constant spacing in the semicircular color pattern. In the central section of the tube, a transverse slow MHD wave is generated that is essentially guided along the field lines. At $t=75 \mathrm{~s}$, we find from Figure $2(\mathrm{a})$ that the wave pattern is confined below the $\beta=1$ surface $(z \sim 0.5 \mathrm{Mm})$. In this region, where the magnetic field can be regarded as weak, the acoustic (fast) mode travels ahead of the (slow) MHD wave (which travels at the Alfvén speed). At the $\beta=1$ level there is a strong coupling between the two modes as previously demonstrated by Rosenthal et al. (2002), Bogdan et al. (2003) and Hasan et al. (2005). Up to this epoch the waves in the individual tubes are sufficiently well separated from each other and the wave pattern in each tube is qualitatively similar to that in a single tube (Hasan \& van Ballegoiijen 2008). However, at $t=122 \mathrm{~s}$ waves emanating from neighboring tubes interact with each other especially in the ambient medium. However, the wave pattern in any tube is not significantly affected by the presence of its neighbors. Furthermore, the slow magneto-acoustic waves above the $\beta=1$ surface are confined close to the central regions of the tubes, where they steepen and produce enhanced heating. This heating appears to be dominantly caused by the wave motions generated at the footpoints and not by the penetration of acoustic waves from the ambient medium or by waves coming from neighboring tubes (Hasan \& van Ballegoiijen 2008).

We now consider the transport of energy in the various wave modes. Following Bogdan et al. (2003) we use the linear expression for the energy flux to identify the energy carried by the dominantly acoustic and magnetic (Poynting flux) components. Table 1 (taken from Vigeesh, Hasan \& Steiner 2008) shows the temporal maximum of the horizontally averaged vertical components of acoustic and Poynting fluxes at three different heights due to impulsive transverse excitation of the lower boundary. They have considered three different amplitudes for the impusive excitations. As seen in Figure 8 of Vigeesh et al. (2008), although the maximum vertical component acoustic fluxes reach values of the order of $10^{7} \mathrm{erg}^{-2} \mathrm{~s}^{-1}$ at a height of $z=1000 \mathrm{~km}$, depending upon the amplitude of the initial excitation, the average fluxes are an order of magintude less.

The Poynting fluxes shown in the table represent the maximum value that the fluxes reach in the interval between the start of the simulation until the time when the fast wave reaches the top boundary (around $60 \mathrm{~s}$ ). Hence these fluxes correspond to the fast mode. The Poynting fluxes associated with the fast mode are relatively lower in magnitude compared to the acoustic fluxes. It should be noted that there is also some Poynting flux associated with the slow mode, since these waves also perturb the magnetic field. But, they are relatively lower than the fluxes that are transported with the fast mode.

Let us now estimate the acoustic energy flux transported into the chromosphere through a single short duration pulse as considered by Vigeesh et al. (2008). The maximum values 

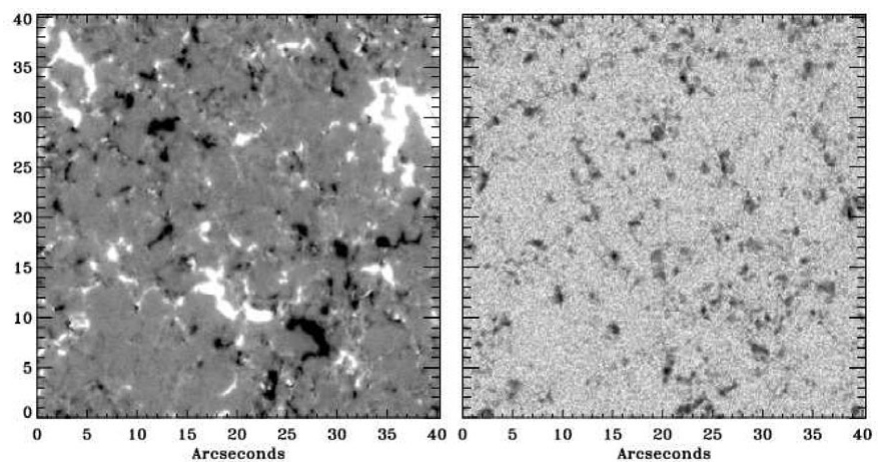

Figure 3. Vertical $B_{\text {app }}^{L}$ (left panel) and horizontal $B_{\text {app }}^{T}$ apparent flux densities in a quiet-Sun map. The grey scale for $B_{\text {app }}^{L}$ saturates at $\pm 50 \mathrm{Mx} \mathrm{cm}^{-2}$ whereas $B_{\text {app }}^{T}$ saturates at $200 \mathrm{Mx}$ $\mathrm{cm}^{-2}$. White in the left panel is positive flux density, and dark in the right corresponds to high values of the transverse apparent flux density (from Lites et al. 2008).

of the acoustic fluxes at $z=1000 \mathrm{~km}$ is $\sim 15 \times 10^{6} \mathrm{erg} \mathrm{cm}^{-2} \mathrm{~s}^{-1}$ which is adequate to balance the radiative losses (of the order of $10^{7} \mathrm{erg} \mathrm{cm}^{-2} \mathrm{~s}^{-1}$ ) in the magnetic network at chromospheric heights. It should be noted that although the fluxes can reach values upto $10^{7} \mathrm{erg} \mathrm{cm}^{-2} \mathrm{~s}^{-1}$, the average values are much less. But multiple excitations can form waves that develop into shocks that follow one another and overtake, increasing the shock strength and thereby increasing the dissipation. Also, in order to be compatible with the observed quasi-steady $\mathrm{Ca}$ emission the injection needs to be in the form of sustained multiple short duration pulses as argued by Hasan \& van Ballegoiijen (2008). Although long-period acoustic waves (with periods of about $5 \mathrm{~min}$.) have been proposed by De Pontieu et al. (2004) as driving spicules, we do not believe that these are responsible for the heating observed in Ca network grains. It should be pointed out that presently there is no observational confirmation for the existence of short-period magneto-acoustic waves in the network. However, SOT on Hinode provides us the possibility to test this hypothesis and ascertain whether such waves can be considered legitimate candidates for heating the magnetized chromophere.

\section{Internetwork magnetism}

Till recently, it was believed that the magnetic field in the interior of supergranule cells was weak with a mean field strength of a few Gauss. This hypothesis was based on low spatial resolution (greater than $1^{\prime \prime}$ ) measurements (e.g., Meunier, Solanki \& Livingston 1998). However, with increase in resolution, it was found that the magnetic flux in quiet regions increases exponentially with spatial resolution from $1 \mathrm{G}$ for $2^{\prime \prime}-3^{\prime \prime}$ to around $20 \mathrm{G}$ at $0.5^{\prime \prime}$ (Sanchez Almeida et al. 2004) though recent observations from Hinode show that the variation is weak up to $0.3^{\prime \prime}$ resolution (Lites et al. 2007). Hanle depolarization signals are consistent with a "hidden" turbulent magnetic field with a typical range of 20-150 G in the internetwork (Trujillo Bueno et al. 2004).

A major finding has taken place recently regarding the nature of magnetic fields in the internetwork (IN). New observations from the Hinode Stokes Polarimeter (SP) (with a spatial resolution of $0.3^{\prime \prime}$ ) reveal the ubiquitous presence of horizontal fields in the range 100-200 G. Figure 3, taken from Lites et al. (2008), shows the vertical (left panel) and horizontal (right panel) components of the apparent magnetic flux densities $B_{\text {app }}^{L}$ and $B_{\text {app }}^{T}$ respectively in a quiet region of the Sun. These observations show that, whereas the 
vertical magnetic field mainly occurs in the intergranular lanes at the network boundaries, the field in the internetwork regions is dominantly horizontal and well separated from the vertical fields. The horizontal fields, with an average value of at least $55 \mathrm{G}$, are located preferentially at the edges of bright granules. Lites et al. (2008) conjecture that these fields might be important in understanding the "hidden" turbulent flux inferred from the Hanle effect. Recently, Steiner et al. (2008) carried out numerical simulations in order to reproduce these observations. They use the line pair of Fe I $630 \mathrm{~nm}$ to synthesize Stokes profiles and derive a ratio of 4.3 between the horizontal and vertical components of the magnetic field, which is reasonably close to the observed value of around 5 found by Lites et al. (2008). Steiner et al. (2008) suggest that the horizontal fields are generated due to flux expulsion.

\section{Spicules}

Despite their discovery over a century ago, spicules are among the least understood phenomena in the chromosphere. They are jets of gas which can be best observed on the limb in the emission lines of $\mathrm{H}_{\alpha}$ or He I. Spicules are believed to originate in the magnetic network, but the field strength and geometry associated with the spicule channel is not well known. It should be noted that the disc counterpart of spicules are mottles. Recently, some progress has been made in this direction as well as on the mechanism that drives spicules. Trujillo Bueno et al. (2005) used the Hanle and Zeeman effects to estimate a field strength of about $10 \mathrm{G}$ at a height of $2000 \mathrm{~km}$ above the photosphere and inclined at about $35^{\circ}$ to the vertical. Tsiropoula and Tziotziou (2004) independently estimated a field strength of $4.1 \mathrm{G}$ in mottles/spicules based on energy arguments.

From an analysis of high resolution images, De Pontieu et al. (2004) inferred a connection between spicules and photospheric p-modes. Although p-mode photospheric oscillations with $5 \mathrm{~min}$. periods are generally evanescent in the upper photosphere, they can leak sufficient energy into the chromosphere. The tunelling becomes particularly effective for waves in inclined magnetic flux tubes because of their higher acoustic cutoff period which can exceed $300 \mathrm{~s}$ above the temperature minimum (De Pontieu et al. 2004). In the chromosphere, these waves form shocks, which drive spicules similar to the model of Hollweg et al. (1982). Using observations from the Solar Optical Telescope on Hinode, De Pontieu et al. (2007a) hypothesized that there are at least two species of spicules: "type-I" spicules which are driven by shock waves as discussed above with time scales of 3-7 min. and "type-II" spicules that are much more dynamic and very thin (width $\sim 100 \mathrm{~km}$ ), have lifetimes of 10-150 s, and seem to be rapidly heated to transition region temperatures, sending material though the chromosphere at speeds of $50-150 \mathrm{~km} \mathrm{~s}^{-1}$. De Pontieu and collaborators suggest that "type-II" spicules may be due to small-scale reconnection events in the chromosphere. De Pontieu et al. (2007b) point out that spicules exhibit transverse motions with velocities of 10 to $25 \mathrm{~km} \mathrm{~s}^{-1}$ which may be the signature of Alfvén waves.

An alternative mechanism for spicules based on a model first proposed by Pikelner (1969) is that they are driven by magnetic reconnection in mixed polarity regions at network boundaries (Wilhelm 2000). This model is still schematic and has not been investigated quantitatively. Oscillations with periods of around $50 \mathrm{~s}$ have also been observed in spicules, which might be due to kink waves excited by the impact of granules on their footpoints (Kukhianidze et al. 2005) or due to Alfvén waves (Hollweg 1982; Kudoh \& Shibata 1999; De Pontieu et al. 2007b). 


\section{Implications for future observations}

This review has attempted to highlight important new developments concerning processes in the magnetized solar chromosphere as well as to point out some of the outstanding problems. Some implications for future ground and space missions are:

(a) Observations as well as simulations reveal that magnetic structures need to be resolved to an accuracy better than $0.1^{\prime \prime}$ on the solar surface. Neither the present missions such as SOHO or TRACE (1" resolution) and Hinode (150 km resolution) nor those in the near future such as SDO have this capability. It is imperative that the next generation of space missions have instruments that can achieve such an accuracy;

(b) Ground-based observations at good sites using adaptive optics can yield a resolution of $1^{\prime \prime}$ or better. However, to simultaneously achieve high, spatial, temporal and spectral resolution, a large aperture $(\geqslant 2-\mathrm{m})$ is required to get a high throughput of photons;

(c) Polarimetric measurements with a high sensitivity (a few Gauss) of the magnetic field and its inclination over a large field of view are needed to understand the magnetic topology of the chromosphere;

(d) In addition, it is important to examine the distribution of magnetic structures in the network and internetwork regions and determine the magnetic filling factor and also geometry of the magnetic canopy accurately;

(e) Spectroscopic measurements in UV using temperature sensitive lines are essential to demarcate the thermal structure of the chromosphere and cross-correlate it with magnetic observations.

\section{References}

Berger, T. E., Rouppe van der Voort, L. H. M., Löfdahl, M. G., Carlsson, M., Fossum, A., Hansteen, V., Marthinussen, E., Title, A., \& Scharmer, G. 2004, A\& $A$, 428, 613

Berger, T. E. \& Title, A. M. 1996, ApJ, 463, 365

Bogdan, T. J., Carlsson, M., Hansteen, V., McMurry, A., Rosenthal, C. S., Johnson, M., PettyPowell, S., Zita, E. J., Stein, R. F., McIntosh, S. W., \& Nordlund, Å. 2003, ApJ, 599, 626

Carlsson, M. \& Bogdan, T. J. 2006, Roy. Soc. Phil. Trans., Series A, Vol. 364, p. 395

Cauzzi, G., Reardon, K. P., Uitenbroek, H., Cavallini, F., Falchi, A., Falciani, R., Janssen, K., Timmele, T., Vecchio, A., \& Wöger, F. 2008, A\& $A, 480,515$

Choudhuri, A. R., Auffret, H., \& Priest, E. R. 1993, Solar Phys., 143, 49

De Pontieu, B., Erdélyi, R., \& James, S. P. 2004, Nature, 430, 536

De Pontieu, B., McIntosh, S., Hansteen, V. H., Carlsson, M., Schrijver, C. J., Tarbell, T. D., Title, A. M., Shine, R. A., Suematsu, Y., Tsuneta, S., Katsukawa, Y., Ichimoto, K., Shimizu, T., \& Nagata, S. 2007a, PASJ, 59, S655

De Pontieu, B., McIntosh, S., Carlsson, M., Hansteen, V. H., Tarbell, T. D., Schrijver, C. J., Title, A. M., Shine, R. A., Tsuneta, S., Katsukawa, Y., Ichimoto, K., Suematsu, Y., Shimizu, T., \& Nagata, S. 2007b, Science, 318, 1574

Gaizauskas, V. 1985, in Chromospheric Diagnostics and Modeling, ed. B. W. Lites (National Solar Observatory: Sunspot, NM), 25

Hasan, S. S. \& Kalkofen, W. 1999, ApJ, 519, 899

Hasan, S. S., Kalkofen, W., \& van Ballegooijen, A. A. 2000, ApJ, 535, L67

Hasan, S. S., Kalkofen, W., van Ballegooijen, A. A., \& Ulmschneider, P. 2003, ApJ, 585, 1138

Hasan, S. S., \& Ulmschneider, P., 2004, A\&A, 422, 1085

Hasan, S. S., van Ballegooijen, A. A., Kalkofen, W., \& Steiner, O. 2005, ApJ, 631, 1270

Hasan, S. S. \& van Ballegooijen, A. A. 2008, ApJ, 680, 1542

Hollweg, J. V., Jackson, S., \& Galloway, D. 1982, Solar Phys., 75, 35

Huang, P., Musielak, Z. E., \& Ulmschneider, P. 1995, A\& A, 297, 579 
Kalkofen, W. 1997, ApJ., 486, L145

Kukhianidze, V., Zaqarashvili, T. V., \& Khutsishvili, E. 2006, A \& A 449, L35

Kosugi, T., Matsuzaki, K., Sakao, T., Shimizu, T., Sone, Y., Tachikawa, S., Hashimoto, T., Minesugi, K., Ohnishi, A., Yamada, T., Tsuneta, S., Hara, H., Ichimoto, K., Suematsu, Y., Shimojo, M., Watanabe, T., Davis, J. M., Hill, L. D., Owens, J. K., Title, A. M., Culhane, J. L., Harra, L., Doschek, G. A., \& Golub, L. 2007, Solar Phys., 243, 3

Kudoh, T. \& Shibata, K. 1999, ApJ, 514, 493

Langangen, Ø., Carlsson, M., \& Rouppe van der Voort, L. 2007, ApJ, 655, 615

Lites, B. W., Rutten, R. J., \& Kalkofen, W. 1993, ApJ, 414, 345

Lites, B., Socas-Navarro, H., Kubo, M., Berger, T. E., Frank, Z., Shine, R. A., Tarbell, T. D., Title, A. M., Ichimoto, K., Katsukawa, Y., Tsuneta, S., Suematsu, Y., Shimizu, T., \& Nagata, S. 2007, PASJ, 59, S571

Lites, B., Kubo, M., Socas-Navarro, H., Berger, T. E., Frank, Z., Shine, R. A., Tarbell, T. D., Title, A. M., Ichimoto, K., Katsukawa, Y., Tsuneta, S., Suematsu, Y., Shimizu, T., \& Nagata, S. 2008, ApJ, 672, 1237

Meunier, N., Solanki, S. K., \& Livingston, W. C. 1998, A\&A, 331, 771

Muller, R. \& Roudier, Th. 1992, Solar Phys., 141, 27

Muller, R., Roudier, Th., Vigneau, J., \& Auffret, H. 1994, A\&SA, 283, 232

Nisenson, P., van Ballegooijen, A. A., de Wijn, A. G., \& Sütterlin, P. 2003, ApJ, 587, 458

Roberts, B. \& Webb, A. R. 1978, Solar Phys., 56, 5

Rosenthal, C. S., Bogdan, T. J., Carlsson, M., Dorch, S. B. F., Hansteen, V., McIntosh, S. W., McMurry, A., Nordlund, Å., \& Stein, R. F. 2002, ApJ, 564, 508

Rouppe van der Voort, L. H. M., Hansteen, V. H., Carlsson, M., et al., 2005, A\&A, 435, 327

Routh, S., Musielak, Z. E., \& Hammer, R., 2007, Solar Phys., 246, 133

Rutten, R. J. \& Uitenbroek, H. 1991, Sol. Phys., 134, 15

Rutten, R. J. 2006, in Solar MHD: Theory and Observations, Proc. NSO Workshop 23, eds. J. Leibacher, H. Uitenbroek, \& R. J. Stein, ASP Conf. Ser. 354 (San Francisco: ASP), p. 276

Rutten, R. J. 2007, in The Physics of Chromospheric Plasmas, eds. P. Heinzel, I. Dorotovic, \& R. J. Rutten, ASP Conf. Ser. 368 (San Francisco: ASP), p. 27

Sánchez Almeida, J., in The Solar-B Mission and the Forefront of Solar Physics, eds. T. Sakurai and T. Sekii, ASP Conf. Ser. 325, (San Francisco: ASP), p. 115

Schaffenberger, W., Wedemeyer-Böhm, S., Steiner, O., \& Freytag, B. 2006, in Solar MHD: Theory and Observations: A High Spatial Resolution Perspective, eds. J. Leibacher, R. F. Stein, and H. Uitenbroek, ASP Conf. Ser. Vol. 354, (San Francisco: ASP), 345

Simon, G. W. \& Leighton, R. B. 1964, ApJ, 140, 1120

Spruit, H. C. 1981, A\&SA, 102, 129

Steiner, O., Knölker, M., \& Schüssler, M. 1994, in Solar Surface Magnetism, eds. R. J. Rutten \& C. J. Schrijver, NATO ASI Ser. C-433, (Dordrecht: Kluwer), p. 441

Steiner, O., Rezaei, R., Schaffenberger, W., \& Wedemeyer-Böhm, S. 2008, ApJ, 680, L85

Tritschler, A., Schmidt, W., Uitenbroek, H., \& Wedemeyer-Böhm, S. 2007, A\&A, 462, 303

Trujillo Bueno, J., Merenda, L., Centeno, R. et al. 2005, ApJ, 619, L191-L194

Tsiropoula, G., \& Tziotziou, K., 2004, A\& A, 424, 279

Ulmschneider, P., Zähringer, K., \& Musielak, Z. E., 1991, A \& A, 241, 625

Ulmschneider, P. 2003, in Lectures on Solar Physics, eds. Antia, H. M., Bhatnagar, A., \& Ulmschneider P., Lecture Notes in Physics 619, Springer Verlag, Heidelberg, Berlin, p. 232

van Ballegooijen, A. A., \& Nisenson, P. 1998, in High Resolution Solar Physics: Theory, Observations, and Techniques, eds. T. R. Rimmele, K. S. Balasubramaniam, \& R. R. Radick, ASP Conf. Ser. 183 (San Francisco: ASP), 30

Vigeesh, G., Hasan, S. S., \& Steiner, O. 2008, A\&A, submitted

Vögler, A., Shelyag, S., Schüssler, M., Cattaneo, F., \& Emonet, T. 2005, A\&6A, 429, 335

Wilhelm, K. 2000, A\&A A, 360, 351

Zhugzhda, Y. D., Bromm, V., \& Ulmschneider, P. 1995, A\&A, 300, 302

Zwaan, C. 1987, ARAA, 25, 83 


\section{Discussion}

ShibATA: I have one comment and one question. My comment is about spicule models. You introduced the reconnection model and the Alfven wave model as different models. However, our numerical simulation (Takenchi and Shibata 2001, ApJ 546, L73) shows that Alfven waves are generated by reconnection. So the reconnection model and the Alfven wave model are not necessarily separate models. Furthermore, slow mode magnetoacoustic shocks are generated from nonlinear Alfven waves by mode coupling. Altogether, slow shocks, Alfven waves, and reconnection are all closely related. My question is also about spicules. I am not convinced about the two types of spicules. What is the fraction of type I and type II spicules?

HASAN: The groups that have introduced this classification of two categories of spicules have, to the best of my information, not provided statistics on this fraction. However, I agree with you that it would be important to know this.

WeBB: We have heard that Hinode sees ubiquitous Alfven waves that dissipate sufficient energy to heat the corona. Have you checked energetics of your shock dissipation model to see if it can provide sufficient energy to heat the corona?

HASAN: As I showed, the Poyting flux that corresponds to the energy flux in the magnetic wave in our simulations is an order of magnitude lower than is required for coronal heating. The important question which needs to be addressed is how this energy can be efficiently dissipated in the corona. 